\title{
Targeting the CALCB/RAMP1 axis inhibits growth of Ewing sarcoma
}

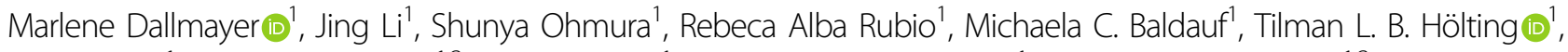
Julian Musa', Max M. L. Knott $\mathbb{B}^{1,2}$, Stefanie Stein', Florencia Cidre-Aranaz $\mathbb{D}^{1}$, Fabienne S. Wehweck ${ }^{1,2}$, Laura Romero-Pérez [1', Julia S. Gerke', Martin F. Orth ${ }^{1}$, Aruna Marchetto', Thomas Kirchner ${ }^{2,3,4}$, Horacio Bach ${ }^{5}$, Giuseppina Sannino ${ }^{1}$ and Thomas G. P. Grünewald ${ }^{1,2,3,4}$

\begin{abstract}
Ewing sarcoma (EwS) is an aggressive cancer characterized by chromosomal translocations generating fusions of the EWSR1 gene with ETS transcription factors (in 85\% FL11). EWSR1-FLI1 induces gene expression via binding to enhancerlike GGAA-microsatellites, whose activity correlates with the number of consecutive GGAA-repeats. Herein we investigate the role of the secretory neuropeptide CALCB (calcitonin-related polypeptide $\beta$ ) in EwS, which signals via the CGRP (calcitonin gene-related peptide) receptor complex, containing RAMP1 (receptor activity modifying protein 1) as crucial part for receptor specificity. Analysis of 2678 gene expression microarrays comprising 50 tumor entities and 71 normal tissue types revealed that CALCB is specifically and highly overexpressed in EwS. Time-course knockdown experiments showed that CALCB expression is tightly linked to that of EWSR1-FLI1. Consistently, gene set enrichment analyses of genes whose expression in primary EwS is correlated to that of CALCB indicated that it is co-expressed with other EWSR1-FLI1 target genes and associated with signatures involved in stemness and proliferation. Chromatin immunoprecipitation followed by sequencing (ChIP-seq) data for FLI1 and histone marks from EwS cell lines demonstrated that EWSR1-FLI1 binds to a GGAA-microsatellite close to CALCB, which exhibits characteristics of an active enhancer. Reporter assays confirmed the strong EWSR1-FLI1- and length-dependent enhancer activity of this GGAAmicrosatellite. Mass spectrometric analyses of EwS cell culture supernatants demonstrated that CALCB is secreted by EwS cells. While short-term RNA interference-mediated CALCB knockdown had no effect on proliferation and clonogenic growth of EwS cells in vitro, its long-term knockdown decreased EwS growth in vitro and in vivo. Similarly, knockdown of RAMP1 reduced clonogenic/spheroidal growth and tumorigenicity, and small-molecule inhibitors directed against the RAMP1-comprising CGRP receptor reduced growth of EwS. Collectively, our findings suggest that CALCB is a direct EWSR1-FLI1 target and that targeting the CALCB/RAMP1 axis may offer a new therapeutic strategy for inhibition of EwS growth.
\end{abstract}

\section{Introduction}

Ewing sarcoma (EwS) is a malignant tumor of bone and soft tissue predominantly affecting children and

\footnotetext{
Correspondence: Thomas G. P. Grünewald

(thomas.gruenewald@med.uni-muenchen.de)

${ }^{1}$ Max-Eder Research Group for Pediatric Sarcoma Biology, Institute of

Pathology of the LMU Munich, Munich, Germany

${ }^{2}$ Institute of Pathology of the LMU Munich, Munich, Germany

Full list of author information is available at the end of the article.

Edited by B. Rotblat
}

adolescents $^{1}$. Since specific treatment options do not exist, current therapy concepts comprise local surgery combined with conventional poly-chemotherapy and irradiation $^{1}$. Despite such intense conventional therapy, prognosis of patients with metastatic disease still remains poor $^{2}$. Thus specific and, in particular, less toxic treatment options are urgently required.

EwS is characterized by gene fusions involving the EWSR1 gene on chromosome 22 (chr22) and various

\section{(c) The Author(s) 2019}

(c) Open Access This article is licensed under a Creative Commons Attribution 4.0 International License, which permits use, sharing, adaptation, distribution and reproduction in any medium or format, as long as you give appropriate credit to the original author(s) and the source, provide a link to the Creative Commons license, and indicate if changes were made. The images or other third party material in this article are included in the article's Creative Commons license, unless indicated otherwise in a credit line to the material. If material is not included in the article's Creative Commons license and your intended use is not permitted by statutory regulation or exceeds the permitted use, you will need to obtain permission directly from the copyright holder. To view a copy of this license, visit http://creativecommons.org/licenses/by/4.0/. 
members of the ETS family of transcription factors-most commonly FLI1 on chr11 (85\% of cases) ${ }^{1}$. EWSR1-FLI1 can arise either through balanced chromosomal translocations or through complex genomic breakage/fusion events $^{3,4}$. Notably, EWSR1-FLI1 encodes an aberrant chimeric transcription factor, which binds DNA at ETSbinding site-like GGAA-motifs and furthermore at GGAA-microsatellites consisting of multiple sequential GGAA-motifs ${ }^{5}$. While EWSR1-FLI1 binding at single ETS binding site-like motifs in gene promoters either activates or represses gene transcription, EWSR1-FLI1 binding at GGAA-microsatellites creates de novo enhancers, whose activity correlates positively with the number of consecutive GGAA-repeats ${ }^{1,6,7}$.

Recent sequencing efforts revealed EWSR1-ETS translocations being virtually the only highly recurrent somatic mutation in $\mathrm{EwS}^{8,9}$. Although EwS is genetically well characterized, its precise cell of origin remains controversial. Transcriptome profiling and functional studies suggested that EwS may arise from mesoderm- or neural crest-derived mesenchymal stem cells ${ }^{10,11}$. Owing to this histogenic uncertainty, there is currently no bona fide genetically engineered animal model available for EwS, which hampers the development of new therapeutic strategies $^{1,12}$. Like many other ligand-independent transcription factor oncoproteins, EWSR1-FLI1 also proved to be notoriously difficult to target ${ }^{1,13}$. However, the EWSR1FLI1-induced transcriptomic signature may harbor specific changes that could be exploited therapeutically.

To explore such EWSR1-FLI1 surrogate targets, we focused in this study on the putative EWSR1-FLI1 target gene $C A L C B$ (calcitonin related polypeptide $\beta$; alias CGRP2, calcitonin gene-related peptide 2), which encodes a neuropeptide that was already described in 1987 to be highly expressed in EwS cell lines ${ }^{14,15}$. Nevertheless, its functional effects in EwS have remained unexplored until now.

The $C A L C B$ gene is located next to its homolog $C A L C A$ (calcitonin related polypeptide $\alpha$ ) on chr11p15.2 and encodes a secretory neuropeptide composed of 37 amino acids $^{16,17}$. CALCB is predominantly expressed in the central nervous system and causes potent vasodilatation ${ }^{18,19}$. Signaling of both CALCA and CALCB is mediated through $G$ protein-coupled receptor complexes present on the cell surface. There is a variety of different receptors, formed by heterodimerization, which recognize both peptides. Most importantly they are recognized by the so called CGRP receptor, which is formed by the calcitonin receptor-like receptor (CLR, encoded by the CALCRL gene) and RAMP1 (receptor activity-modifying protein 1). RAMP1 makes the receptor complex specific for the binding of CALCA and $\mathrm{CALCB}^{20,21}$. Receptor-ligand interaction leads to $\mathrm{G}$ protein-mediated increase in intracellular cAMP levels ${ }^{22}$. Apart from the above-described CGRP receptor, CALCB also binds to a receptor complex consisting of RAMP1 and the calcitonin receptor (CTR, encoded by the CALCR gene), which is called $\mathrm{AMY}_{1}$ (amylin subtype 1) receptor. However, this receptor is not specific for CALCA and CALCB but is also activated by binding of islet amyloid polypeptide (IAPP). Since the biological role of $\mathrm{AMY}_{1}$ is not fully understood, and given that both CALCR and IAPP are not or only barely expressed in EwS (Supplementary Figure S1), we focused in this study on CALCB and the CGRP receptor containing CLR and RAMP1 ${ }^{21}$.

Here we show that $C A L C B$ is an EWSR1-FLI1 target gene highly overexpressed in EwS as compared to normal tissues and other childhood malignancies and that its high expression is likely mediated through EWSR1-FLI1 binding to an enhancer-like GGAA-microsatellite. Proteomic and functional analyses revealed that CALCB, but not CALCA, is secreted by EwS cells and that suppression of either $C A L C B$ or its receptor's component RAMP1 significantly reduced proliferation and clonogenic/spheroidal growth of EwS cells in vitro, as well as tumor growth in vivo, which can be mimicked in vitro by application of the small molecule CGRP receptor inhibitors MK-3207 and BIBN-4096 (Olcegepant).

\section{Materials and methods \\ Analysis of microarray data}

The microarray datasets for cancer and normal tissues were downloaded from public repositories and processed as described previously ${ }^{23}$. Data generated on Affymetrix HG-U133Plus2.0 microarrays were normalized simultaneously by Robust Multi-chip Average (RMA) using brainarray chip description files (CDF; ENTREZg, v21) yielding one optimized probe-set per gene ${ }^{24,25}$. Accession codes of used datasets are given in Supplementary Table 1.

\section{Cell culture and provenience of cell lines}

A673, HEK-293T, and SK-PN-DW cells were purchased from the American Type Culture Collection (ATCC, Manassas, VA, USA; CRL-1598, CRL-1573, and CRL2139, respectively). RDES, SK-ES1, SK-N-MC, and MHHES1 cells were provided by the German Collection of Microorganisms and Cell Cultures (DSMZ, Braunschweig, Germany). TC-71 cells were kindly provided by the Children's Oncology Group and ES7, EW-1, EW-3, EW-7, EW-16, EW-18, EW-22, EW-24, LAP35, MIC, ORS, POE, SKNPLI, and STA-ET1 cells were provided by O. Delattre (Institute Curie, Paris, France). SB-KMS-KS1 was established in the Department of Pediatrics at the TU Munich (Munich, Germany) and described previously ${ }^{26}$. A673/ TR/shEF1 cells, which contain a doxycycline (dox)-inducible short hairpin RNA (shRNA) against EWSR1-FLI1, were kindly provided by J. Alonso (Madrid, Spain ${ }^{27}$. All cell lines were grown at $37^{\circ} \mathrm{C}$ and $5 \% \mathrm{CO}_{2}$ in a humidified atmosphere. RPMI 1640 medium with stable glutamine (Biochrom, Berlin, Germany), 10\% tetracycline-free fetal 
calf serum (FCS; Biochrom), $100 \mathrm{U} / \mathrm{ml}$ penicillin (Biochrom), and $100 \mu \mathrm{g} / \mathrm{ml}$ streptomycin (Biochrom) was used as standard medium to grow the cells. For cell lines, which tend to grow in suspension, TPP cell culture flasks (Faust, Klettgau, Germany) were coated with 1:40 phosphate-buffered saline (PBS)-diluted (Biochrom) collagen solution (Sigma-Aldrich/Merck Millipore, Darmstadt, Germany) to enable adherent growth. Cells were routinely checked for mycoplasma infection by nested PCR. Cell line purity was confirmed by Short Tandem Repeat profiling.

\section{RNA extraction, reverse transcription, and quantitative real-time PCR (qRT-PCR)}

RNA for analysis of gene expression with qRT-PCR from cell lysates and frozen tumor tissue was extracted using the NucleoSpin RNA Kit (Macherey-Nagel, Düren, Germany). Subsequent reverse transcription was performed with the High Capacity cDNA Reverse Transcription Kit (Thermo Fisher Scientific, Waltham, MA, USA) utilizing $1 \mu \mathrm{g}$ RNA per reaction and following the manufacturers' protocols of both kits. qRT-PCRs were performed using SYBR green (Applied Biosystems, Waltham, MA, USA) with a total volume of $15 \mu \mathrm{l}$. cDNA was diluted $1: 10$ and concentration of primers was $0.5 \mu \mathrm{M}$. Oligonucleotides were purchased from MWG Eurofins Genomics (Ebersberg, Germany). Expression levels were determined with the CFX Connect Real time PCR Cycler (Bio-Rad Laboratories, Hercules, CA, USA) in a two-step protocol: initial enzyme activation and denaturation at $95^{\circ} \mathrm{C}$ for $2 \mathrm{~min}$, denaturation at $95^{\circ} \mathrm{C}$ for $10 \mathrm{~s}$, and annealing and extension at $60^{\circ} \mathrm{C}$ for $20 \mathrm{~s}$ (repeating the last two steps 49 times), followed by a melting curve starting at $55^{\circ} \mathrm{C}$ and increasing by $0.5^{\circ} \mathrm{C}$ every $10 \mathrm{~s}$ until a temperature of $95^{\circ} \mathrm{C}$ was reached. Expression levels were calculated according to the $2^{-\Delta \Delta C T}$ method $^{28}$. RPLPO served as housekeeping gene. Primer sequences were as follows:

RPLPO forward, 5'-GAAACTCTGCATTCTCGCTTC-3'; RPLPO reverse, 5'-GGTGTAATCCGTCTCCACAG-3';

EWSR1-FLI1 forward, 5'-GCCAAGCTCCAAGTCAAT ATAGC-3';

EWSR1-FLI1 reverse, 5'-GAGGCCAGAATTCATGTT ATTGC-3';

CALCB forward, 5'-GCTCTCAGTATCTTGGTCCTG-3'; CALCB reverse, 5'-CACATAGTCCTGCACCAGTG-3'; RAMP1 forward, 5'-CCCAGTTCCAGGTAGACATG-3'; RAMP1 reverse, 5'-CCAGCTTCTCCGCCATGTG-3'.

\section{Quantification of $C A L C B$ gene expression levels in EwS cell lines}

Different EwS cell lines were cultured under standard conditions. After a minimum of $48 \mathrm{~h}$ of culture, cells were harvested at a confluency of approximately $80 \%$, and RNA extraction was carried out. $C A L C B$ expression was determined using qRT-PCR as described. $C A L C B$ expression levels were calculated relative to that of the A673 EwS cell line.

\section{Quantification of EWSR1-FLI1-dependent CALCB gene expression in vivo}

For analysis of in vivo CALCB expression dependence on EWSR1-FLI1, $5 \times 10^{6}$ A673/TR/shEF1 EwS cells, which harbor a dox-inducible shRNA against EWSR1FLI1, were injected subcutaneously in the flanks of immunocompromised NSG (Nod/scid/gamma) mice. When tumors reached an average volume of $180 \mathrm{~mm}^{3}$, mice were randomized and either received $2 \mathrm{mg} / \mathrm{ml}$ dox (Beladox, bela-pharm, Vechta, Germany) and 5\% sucrose (Sigma-Aldrich/Merck Millipore) in the drinking water $(\mathrm{dox}+)$ or only $5 \%$ sucrose (dox-). Mice were sacrificed $96 \mathrm{~h}$ after beginning of dox treatment, and tumors were collected for RNA analysis. Total RNA was extracted using the ReliaPrep miRNA Cell and Tissue Miniprep System (Promega, Madison, WI, USA). Knockdown of EWSR1-FLI1 was confirmed by qRT-PCR and proved EWSR1-FLI1 expression to be downregulated to $15 \%$ of the control (dox-). Tumor purity (>95\%) was confirmed in routine histology (hematoxylin and eosin [H\&E] stains). The transcriptomes of 3 tumors of each group were profiled on Affymetrix Clariom D arrays (RNA integrity number $>9$ ). Microarray data were simultaneously normalized on gene level using Signal Space Transformation RMA and Affymetrix CDF as described ${ }^{29}$.

\section{Analysis of chromatin-immunoprecipitation followed by sequencing (ChIP-Seq) data}

Publicly available data were retrieved from the Gene Expression Omnibus (GEO; GSE61944) ${ }^{7}$ and the ENCODE project $^{30}$, processed as described ${ }^{31}$, and displayed in the UCSC genome browser. The used samples are available under the following accession codes:

GSM736570 ENCODE_SKNMC_hg19_DNAseHS_rep2;

GSM1517546 SKNMC.shGFP96.FLI1;

GSM1517555 SKNMC.shFLI196.FLI1;

GSM1517548 SK-N-MC_shGFP_96h_H3K4me1;

GSM1517557 SK-N-MC_shFLI1_96h_H3K4me1;

GSM1517547 SKNMC.shGFP96.H3K27ac;

GSM1517556 SKNMC.shFLI196.H3K27ac;

GSM1517569 A673.shGFP48.FLI1;

GSM1517572 A673.shFLI148.FLI1;

GSM1517571 A673.shGFP96.H3.k27ac;

GSM1517574 A673.shFLI196.H3K27ac.

\section{Dual luciferase reporter assays}

A 359 base pairs (bp) fragment around the $C A L C B$ associated GGAA-microsatellite was cloned from two different EwS cell lines (TC-71 and MHH-ES1) by PCR 
into the pGL3 Luciferase Reporter Vector (Promega) using the In-Fusion HD Cloning Kit (Clontech, Takara Bio USA, CA, USA). The DNA from the cell lines was extracted using the NucleoSpin Tissue genomic DNA Prep Kit (Macherey-Nagel) and digested with the restriction enzymes Eco-RV and SphI (New England Biolabs [NEB], Ipswich, MA, USA), followed by a fragment separation by gel electrophoresis, and fragment purification of the band at $8200 \mathrm{bp}$ using the NucleoSpin Gel and PCR Clean-up Kit (Macherey-Nagel). A touchdown PCR was performed with $100 \mathrm{ng}$ of the purified DNA fragment using the Infusion-Primers:

forward: 5'-ctagccegggctcgagGAGCCCTTTAGTATCC CCTTTG-3';

reverse: 5'-gatcgcagatctcgagACCCTTGTACTAACATG CTTCG-3',

(MWG Eurofins Genomics) at a concentration of 0.5 $\mu \mathrm{M}$, and the components of the Go Taq Hot Start Polymerase Kit (Promega) according to the manufacturer's instructions. The thermal protocol was as follows: activation at $95^{\circ} \mathrm{C}$ for $2 \mathrm{~min}$, followed by 20 cycles of $98^{\circ} \mathrm{C}$ for $10 \mathrm{~s}, 59-49^{\circ} \mathrm{C}$ for $30 \mathrm{~s}$ (decreasing $0.5^{\circ} \mathrm{C}$ in each cycle), and $72{ }^{\circ} \mathrm{C}$ for $1 \mathrm{~min}$, finalizing at $72^{\circ} \mathrm{C}$ for $5 \mathrm{~min}$. The PCR product was separated by gel electrophoresis, and the desired fragment of $359 \mathrm{bp}$ was purified with the NucleoSpin Gel and PCR Clean-up Kit (Macherey-Nagel). The reaction with the In-Fusion HD Cloning Kit (Clontech) was initiated using $10 \mathrm{ng}$ of the linearized pGL3 Luciferase Reporter Vector (Promega), digested with XhoI (NEB) and prepared according to the manual, and $20 \mathrm{ng}$ of the purified DNA fragment. The reaction was incubated at $50^{\circ} \mathrm{C}$ for $15 \mathrm{~min}$, stopped on ice for $5 \mathrm{~min}$, and Escherichia coli Stellar competent cells (Clontech) were transformed with the DNA of the infusion mixture. Bacteria were grown on agar plates supplemented with ampicillin at a final concentration of $100 \mu \mathrm{g} / \mathrm{ml}$ (SigmaAldrich/Merck Millipore) overnight at $37^{\circ} \mathrm{C}$. Colonies were picked the next morning and checked for clones containing the plasmid with the insert by colony PCR. Positive clones were incubated overnight in LB broth (Miller)-medium with $100 \mu \mathrm{g} / \mathrm{ml}$ ampicillin (SigmaAldrich/Merck Millipore) at $37^{\circ} \mathrm{C}$, and plasmids were purified using the PureYield Plasmid Midiprep System 2 (Promega). The identity of the fragment was validated by Sanger sequencing (Eurofins GATC Biotech, Konstanz, Germany).

For the luciferase reporter assay, $2 \times 10^{5}$ A673/TR/ shEF1 EwS cells, harboring a dox-inducible shRNA against EWSR1-FLI1, were plated in a well of a 6-well plate (TPP, Faust) in $1.8 \mathrm{ml}$ of growth medium and transfected with the microsatellite-containing pGL3-luc vector and Renilla pGL3-Rluc vector (ratio 100:1) using Lipofectamine LTX with Plus Reagent (Thermo Fisher Scientific). Transfection medium was replaced by media with/without dox $(1 \mu \mathrm{g} / \mathrm{ml}$; VWR/Merck, Radnor, PA, USA) $4 \mathrm{~h}$ after transfection. After $72 \mathrm{~h}$, the cells were lysed and assayed with a dual luciferase assay system (Berthold Technologies, Bad Wildbad, Germany). Firefly luciferase activity was normalized to Renilla luciferase activity.

\section{Gene set enrichment analysis (GSEA)}

To identify gene signatures and biological processes associated with $C A L C B$ in normalized gene expression data from 166 primary EwS ${ }^{32}$, GSEA was performed on lists of genes ranked by their correlation coefficient with $C A L C B$ (MSigDB, c2.cpg.v6.2). GSEA was carried out with 1000 permutations in default settings ${ }^{33}$.

\section{Generation of cell lines with dox-inducible shRNAs}

For generation of EwS cell lines with dox-inducible constructs (here in A673 and RDES), either a nontargeting negative control shRNA (MWG Eurofins Genomics) or specific shRNAs targeting $C A L C B$ or RAMP1 (MWG Eurofins Genomics) were cloned in the pLKO-Tet-on-all-in-one system (Addgene plasmid \# 21915, Cambridge, MA, USA) as described previously ${ }^{34}$. Lentivirus production was performed in HEK-293T cells. A673 and RDES EwS cells were infected with the respective lentiviruses and selected with $1.5 \mu \mathrm{g} / \mathrm{ml}$ puromycin (Invivogen, Toulouse, France). Single-cell cloning was performed, and knockdown efficacy of individual clones was assessed by qRT-PCR $48 \mathrm{~h}$ after addition of dox $(1 \mu \mathrm{g} / \mathrm{ml}$; VWR/Merck). The shRNA target sequences were as follows:

shControl, 5'-CAACAAGATGAAGAGCACCAA-3'; shCALCB1, 5'-AAGGAATGAAACTGAATGCAA-3'; shCALCB4, 5'-AACCTTGGTGATGCATTACAA-3'; shRAMP1_3, 5'-GCGCACTGAGGGCATTGTGTA-3'; shRAMP1_4, 5'-TGCCTGCCAGGAGGCTAACTA-3'.

\section{Proliferation assays}

A total of $1-5 \times 10^{5}$ cells were seeded in 6-well plates (TPP, Faust) in $1.5 \mathrm{ml}$ of standard growth medium. Gene knockdown was induced by addition of $1 \mu \mathrm{g} / \mathrm{ml}$ dox (VWR/Merck) to the growth medium (refreshed every 48-72 h) of cells harboring an inducible shRNA against $C A L C B$ or by serial transfections with $25 \mathrm{nM}$ small interfering RNA (siRNA) directed against $C A L C B$ (Hs_CALCB_1 FlexiTube siRNA or Hs_CALCB_4 FlexiTube siRNA, QIAGEN, Hilden, Germany) or a scrambled control siRNA (MISSION siRNA Universal Negative Control \#1, Sigma-Aldrich/Merck Millipore) following the manufacturer's handbook of the transfection reagent HiPerfect (QIAGEN). After $2-4 \mathrm{~h}, 3 \mathrm{ml}$ of standard growth medium was added to prevent toxic effects of the transfection reagent. After $24 \mathrm{~h}$, the medium was exchanged and after another $24 \mathrm{~h}$ a second transfection was performed. Cell counts were determined by using 
standardized hemocytometers (C-chips, Biochrom) and Trypan-blue (Sigma-Aldrich/Merck Millipore) exclusion $72 \mathrm{~h}$ after seeding in the short-term proliferation assay and 6-9 days after seeding in the long-term proliferation assay.

\section{Colony-forming assays}

A673 and RDES EwS cells harboring a dox-inducible shRNA construct against $C A L C B$ or RAMP1 or RDES wild-type cells were seeded at concentrations of 100-1 000 cells per well of 12-well plates and grown in standard culture medium for 12-14 days. Cells were treated with/ without $1 \mu \mathrm{g} / \mathrm{ml}$ dox (VWR/Merck) and RDES wild-type cells were serially transfected with a non-targeting siRNA or siRNAs against $C A L C B$ (as described above). Twice gently PBS-washed (Biochrom) colonies were stained with $500 \mu \mathrm{l}$ crystal violet (Sigma-Aldrich/Merck Millipore) and the number of colonies was quantified using ImageJ.

\section{Sphere-formation assays}

For analysis of three-dimensional (3D) sphere formation, A673 and RDES EwS cells harboring a dox-inducible shRNA construct against $C A L C B$ or RAMP1 were seeded at a density of 1000 cells per well of ultra-low attachment 96well plates (Corning, NY, USA) in $80 \mu \mathrm{l}$ standard cell culture medium with/without dox $(1 \mu \mathrm{g} / \mathrm{ml}$; VWR/ Merck). The culture medium was refreshed by adding $10 \mu \mathrm{l}$ medium with/without dox on top every second day. Spheroidal growth was monitored for 14 days. Thereafter, phase-contrast imaging and morphological analyses of spheres were carried out with an inverted Zeiss Axiovert 25 microscope (Jena, Germany) equipped with a Zeiss Axiocam 105 color camera (Aptina CMOS Color Sensor, square pixels of $2.2 \mu \mathrm{m}$ side length, $2560 \times 1920$ pixel resolution). Sphere numbers and diameters were analyzed with ImageJ.

\section{Analysis of tumor growth in vivo}

A total of $2.5 \times 10^{6}$ A673 EwS cells harboring a doxinducible shRNA construct against $C A L C B$ or RAMP1 or a non-targeting control shRNA (shControl) were injected subcutaneously in NSG mice. After 10-14 days, when tumors were first palpable, mice were randomized and thereafter received either $2 \mathrm{mg} / \mathrm{ml}$ dox (bela-pharm) dissolved in sterile water containing 5\% sucrose (SigmaAldrich/Merck Millipore) (dox+) or sterile water with 5\% sucrose alone (dox-). Tumor growth was monitored with a caliper every other day and mice were sacrificed by cervical dislocation when the tumors exceeded an average diameter of $15 \mathrm{~mm}$ (prior start of the experiment defined as "event"). Experiments were approved by the government of Upper Bavaria and conducted in accordance with ARRIVE guidelines and recommendations of the European Community (86/609/EEC) and UKCCCR (guidelines for the welfare and use of animals in cancer research).

\section{Small-molecule inhibitor assays}

A673 and A673/TR/shRAMP1_4 EwS cells, the latter harboring a dox-inducible shRNA against RAMP1, were seeded at a density of 1500 cells per well of a 96-well plate (TPP, Faust) in $50 \mu \mathrm{l}$ standard growth medium with/ without dox ( $1 \mu \mathrm{g} / \mathrm{ml}$; VWR/Merck). After $24 \mathrm{~h}$ of incubation, treatment was started by addition of $50 \mu \mathrm{l}$ standard growth medium containing either different concentrations of the CGRP receptor inhibitor MK-3207 (AdooQ Bioscience, Irvine, CA, USA) dissolved in dimethyl sulfoxide (DMSO; Sigma-Aldrich/Merck Millipore) or the corresponding concentration of DMSO alone and dox refreshment for dox + wells. After $72 \mathrm{~h}$, read-out was performed by addition of $20 \mu \mathrm{l}$ of 1:10 dissolved Resazurin ( $1 \mathrm{~g} / \mathrm{l}$, Sigma-Aldrich/Merck Millipore) to the cells and measurement of fluorescence with a plate reader (Thermo Fisher Scientific) after $7 \mathrm{~h}$ of incubation.

For analysis of two-dimensional (2D) colony-formation capacity under inhibitor treatment, A673 and RDES EwS cells were seeded at a density of 100 cells per well of 12-well plates (TPP, Faust) in $1 \mathrm{ml}$ culture medium. Forty-eight hours after seeding, inhibitors were added at final concentrations of $100 \mu \mathrm{M}$ for BIBN-4096 (Olcegepant; R\&D systems, Minneapolis, MN, USA) and $20 \mu \mathrm{M}$ for MK-3207 (AdooQ Bioscience). DMSO (Sigma-Aldrich/Merck Millipore) served as control. After 1-3 weeks, colonies were gently washed twice with PBS (Biochrom) and stained with $500 \mu \mathrm{l}$ crystal violet solution (Sigma-Aldrich/Merck Millipore). Colonies were photographed, and the number of colonies was counted using Image J.

For analysis of 3D sphere-formation capacity under inhibitor treatment, A673 and RDES EwS cells were seeded at a density of 1000 cells per well in $80 \mu \mathrm{l}$ culture medium in wells of 96-well ultra-low attachment culture plates (Corning). After $24 \mathrm{~h}$ of incubation, $20 \mu \mathrm{l}$ culture medium containing either inhibitor or DSMO (control; Sigma-Aldrich/Merck Millipore) was added to the wells resulting in a final concentration of $100 \mu \mathrm{M}$ BIBN-4096 (Olcegepant; R\&D systems) or $20 \mu \mathrm{M}$ MK-3207 (AdooQ Bioscience), respectively. After 14 days, spheres were photographed, and their number and size were analyzed using ImageJ.

\section{Human samples and immunohistochemistry (IHC)}

Available tissue microarrays (TMA) of primary EwS tumors containing 2 cores of each sample, with a diameter of $1 \mathrm{~mm}$, as well as internal controls were stained for CALCB. Analysis were carried out with approval from LMU Munich ethics committee.

For IHC, 4- $\mu \mathrm{m}$ sections were cut and antigen retrieval was carried out by heat treatment using target unmasking fluid (PanPath, Budel, Netherlands). Slides were incubated for $60 \mathrm{~min}$ at room temperature with a rabbit polyclonal anti-CALCB antibody (bs-0791R, Bioss Antibodies Inc., 
MA, USA; dilution 1:120). Then slides were incubated with a secondary anti-rabbit IgG antibody (Vectastain ABC-Kit Elite Universal, Vector laboratories, Burlingame, CA, USA) followed by target detection using DAB plus (Agilent Technologies, Santa Clara, CA, USA). Counterstaining was performed with Hematoxylin Gill's Formula (Vector). Intensity of CALCB staining was scored independently by two researchers on a scale from 0 to $2(0=$ majority of the cells is negative for CALCB staining, $1=$ majority of the cells shows moderate CALCB staining, and $2=$ majority of the cells shows strong CALCB staining). Specificity of the anti-CALCB-antibody was assured by determination of immunoreactivity scores (IRSs) using the Remmele and Stegner scoring system ${ }^{35}$ in 6-11 representative high-power fields $(\times 40)$ per xenograft with/without qRT-PCRconfirmed knockdown of $C A L C B$.

\section{CD31 staining and evaluation of microvessel density}

For CD31 staining, 4- $\mu \mathrm{m}$ sections of formalin-fixed and paraffin-embedded tumor tissue derived from EwS xenografts in mice were cut and heat treated using the Target Retrieval Solution (Agilent Technologies). Thereafter, tissue slides were stained with a primary monoclonal rat antiCD31-antibody (DIA-310, Dianova, Hamburg, Germany; dilution 1:150, $60 \mathrm{~min}$ incubation at room temperature). As secondary antibody, a biotinylated and mouse-absorbed anti-rat-IgG-antibody (BA 4001, Vector; dilution 1:100) was used. After Streptavidin horseradish peroxidase (Novocastra Laboratories, Newcastle upon Tyne, United Kingdom) treatment, DAB plus (Agilent Technologies) was used for target detection. The slides were counterstained with Hematoxylin Gill's Formula (Vector).

For evaluation of the microvessel density in the CD31stained slides, the Chalkley-grid method was used ${ }^{36}$. To this end, the number of overlaps of a CD31-positive cell with a dot of the Chalkley-grid in each quarter of the grid in four independent regions of the CD31-stained slide was counted, and the mean vessel density of the tumor was extrapolated.

\section{Evaluation of mitosis and necrosis in xenografts}

The average number of mitotic cells per high-power field was determined in 22 representative A673/TR/ shCALCB xenografts and 10 A673/TR/shRAMP1 xenografts in H\&E-stained slides with/without knockdown of $C A L C B$ or $R A M P 1$, respectively. The average area of necrotic tissue over the total tissue area as well as the number of mitoses per tumor sample was determined by a data-blinded resident pathologist through evaluation of 10 high-power fields $(\times 40)$ per slide.

\section{Mass spectrometric analyses}

A673 EwS cells were seeded at a density of $4 \times 10^{6}$ cells per T150 flask (TPP, Faust) in $20 \mathrm{ml}$ of standard culture medium. After $48 \mathrm{~h}$, the supernatant from the cells was removed, and the cells were washed with PBS (Biochrom) twice. Thereafter, the cells were grown for further $24 \mathrm{~h}$ in $20 \mathrm{ml}$ Opti-MEM (Thermo Fisher Scientific) only. Afterwards, the supernatants were collected and immediately frozen at $-80^{\circ} \mathrm{C}$ until the mass spectrometry was performed at the Antibody Engineering and Proteomics facility of the Immunity and Infection Research Centre (Jack Bell Bldg., Vancouver, Canada). For mass spectrometric analysis, samples were lyophilized and resuspended in $50 \mathrm{mM}$ ammonium bicarbonate. In total $200 \mu \mathrm{g}$ of protein was reduced and alkylated using $10 \mathrm{mM}$ dithiothreitol (Thermo Fisher Scientific) and $100 \mathrm{mM}$ iodoacetamide (Sigma-Aldrich/Merck Millipore), respectively. Next, samples were digested using $20 \mathrm{ng} / \mu \mathrm{l}$ trypsin (NEB) for $18 \mathrm{~h}$ at $37^{\circ} \mathrm{C}$. Samples were separated in a Nano-HPLC (NanoLC-2D, Eksigent, Sciex, CA, USA) using a $\mathrm{C} 18$ column and a gradient composed of solvent $\mathrm{A}$ (5\% acetonitrile) and solvent B (95\% acetonitrile). The program was: $5 \%$ acetonitrile for $5 \mathrm{~min}, 5-100 \%$ for 50 min, and $100 \%$ for $10 \mathrm{~min}$. Eluted samples were spotted (Eksigent) on a 384-well plate and $1 \mu \mathrm{l}$ of the matrix $\alpha$-cyano-4-hydroxycinnamic acid $(10 \mathrm{mg} / \mathrm{ml}$ in $50 \%$ acetonitrile and $0.1 \%$ trifluoroacetic acid) was added. The mass spectrometric analysis was performed on a MALDI-TOF/TOF 4800 (Sciex) using positive mode. The data were analyzed with the Trans-Proteomic Pipeline (Seattle Proteome Center, WA, USA). To identify the peptide profile, a full-length synthetic CALCB polypeptide (Peptides \& Elephants, Henningsdorf, Germany) was processed as a standard and analyzed. The peptide 106SNFVPTNVGSK116 ( $\mathrm{m} / \mathrm{z}$ 1149.5898, monoisotopic) was used to identify the presence of CALCB in the samples.

\section{Results \\ CALCB is an EWSR1-FLI1 target gene highly but heterogeneously expressed in EwS}

In the search of potential EWSR1-FLI1 surrogate targets, we analyzed publicly available gene expression microarray data comprising 71 normal tissue types and 50 tumor entities. Thereby, we identified $C A L C B$ as being highly overexpressed in EwS compared to most tumor entities and all normal tissues except for trigeminal ganglia (Fig. 1a, b; Supplementary Fig. S2). The high expression of CALCB in EwS was validated by IHC staining of a TMA of primary EwS tumors, of which 44\% (39/89) displayed a high and $37 \%$ (33/89) an intermediate IRS (2 or 1, respectively) for CALCB expression (Supplementary Fig. S3).

This EwS-specific expression pattern suggested a potential regulatory relationship between EwS-specific EWSR1ETS fusion oncogenes and CALCB. To test this hypothesis, we performed time-course knockdown experiments of EWSR1-FLI1 in the EwS cell line A673/TR/shEF1, which 


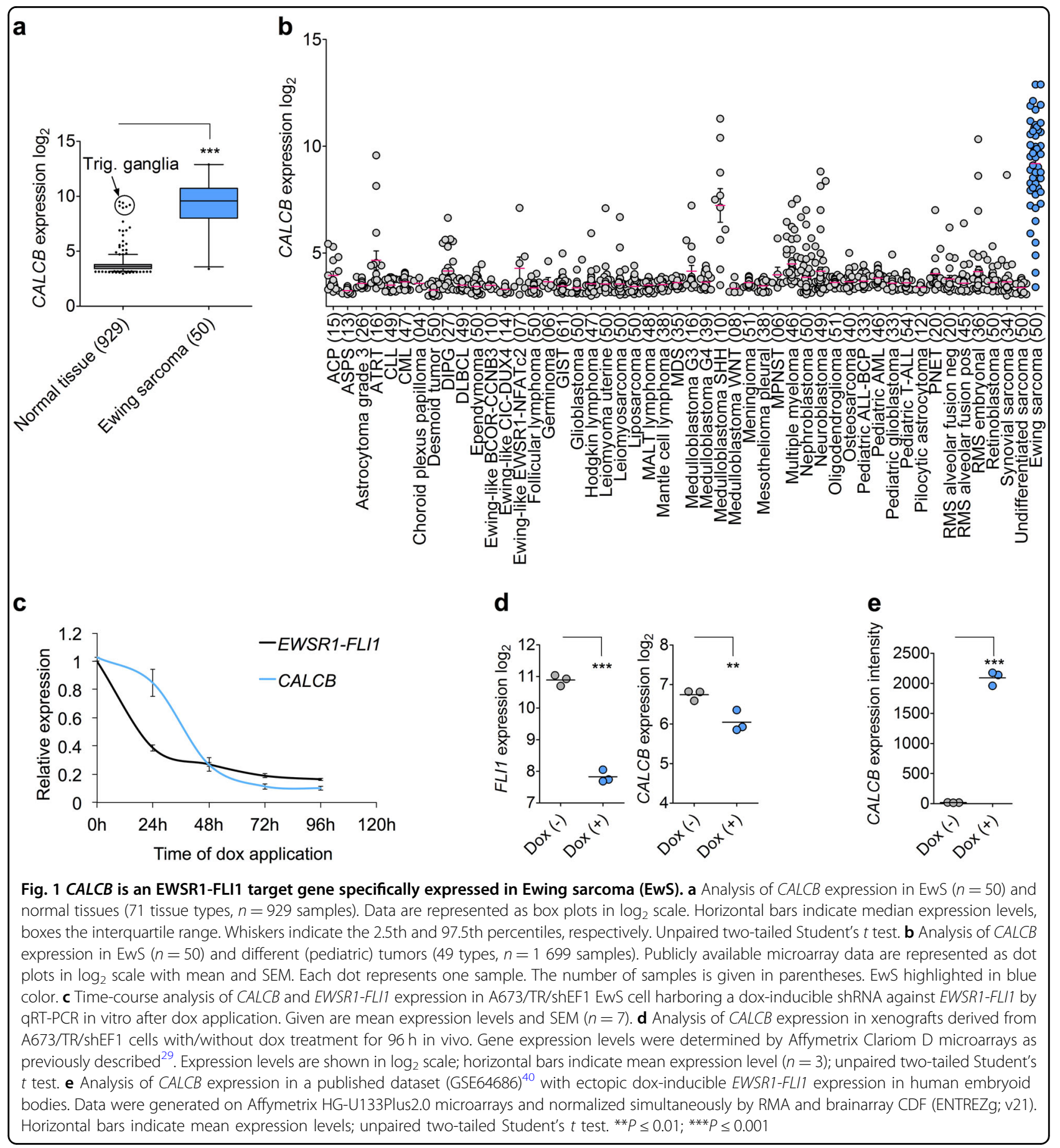

harbors a dox-inducible shRNA against EWSR1-FLI1 and measured the expression of EWSR1-FLI1 and CALCB at different time points after start of dox treatment $(0-96 \mathrm{~h})$ by qRT-PCR. The results showed that the expression of $C A L C B$ is tightly linked to that of EWSR1-FLI1 (Fig. 1c), which was confirmed in xenografts derived from A673/TR/ shEF1 cells in vivo (Fig. 1d). Conversely, ectopic expression of EWSR1-FLI1 in human embryoid bodies strongly induced $C A L C B$ expression (Fig. 1e).
To further assess this regulatory relationship, we explored available FLI1 ChIP-seq data from two EwS cell lines (A673 and SK-N-MC) and found strong EWSR1-FLI1 binding at intron 5 of the longest isoform (isoform 3) of the CALCB gene, which mapped to a GGAA-microsatellite that showed epigenetic characteristics of an active enhancer (Fig. 2a). Knockdown of EWSR1-FLI1 in both cell lines abolished the EWSR1-FLI1 signal at this GGAA-microsatellite and markedly reduced the signals for acetylated H3K27 

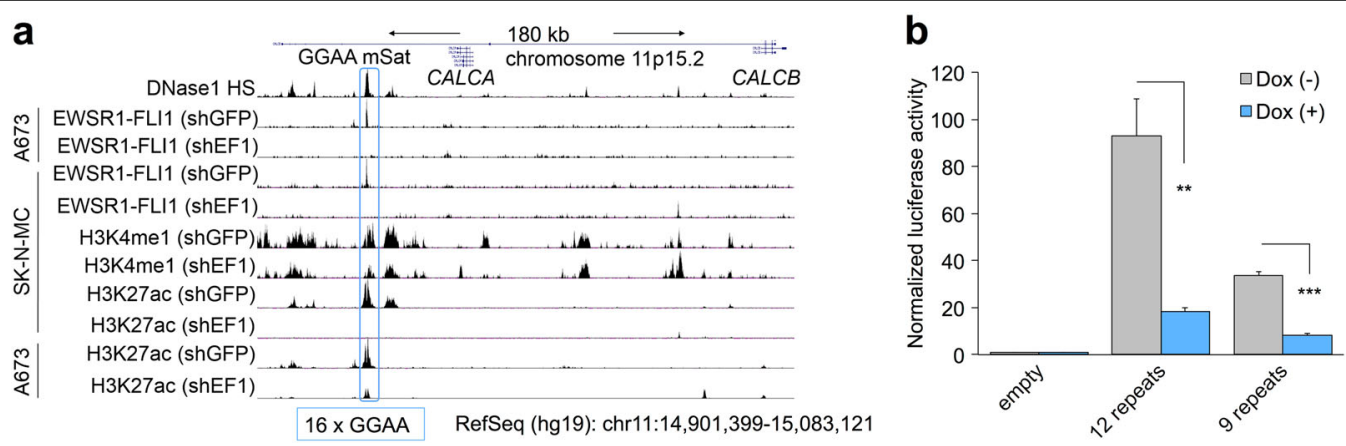

Fig. 2 CALCB expression is regulated through EWSR1-FLI1 binding to a nearby enhancer-like GGAA-microsatellite. a Integrative genomic view of published ChIP-seq ${ }^{7}$ and DNAse-seq data ${ }^{30}$ of the CALCB locus. Data were generated in A673 and SK-N-MC Ewing sarcoma cells, stably transfected with either shRNA targeting GFP (shGFP; negative control) or EWSR1-FLI1 (shEF1). The blue box marks the location of the CALCB geneassociated GGAA-microsatellite, consisting of 16 repeats of the GGAA-motif in the human reference genome (hg19). $\mathbf{b}$ Luciferase reporter assays in A673/TR/shEF1 cells with/without knockdown of EWSR1-FL11 (Dox+/-) $72 \mathrm{~h}$ after transfection with plasmids containing a 359-bp fragment around the CALCB-associated GGAA-microsatellite as displayed in $\mathbf{a}$. Data are presented as mean $(n=3-5)$ and SEM; unpaired two-tailed Student's $t$ test. ${ }^{* *} P \leq 0.01 ;{ }^{* * *} P \leq 0.001$

(H3K27ac), indicating abrogated enhancer activity upon EWSR1-FLI1 silencing (Fig. 2a).

To confirm its EWSR1-FLI1-dependent enhancer activity, we cloned a 359-bp fragment containing this GGAA-microsatellite from two EwS cell lines (TC-71 and MHH-ES1) into the pGL3 luciferase reporter vector and performed reporter assays in A673/TR/shEF1 cells with/ without silencing of EWSR1-FLI1. In these assays, we observed strong enhancer activity of the GGAA-microsatellite, which was significantly diminished upon EWSR1FLI1 knockdown (Fig. 2b). In accordance with the higher number of consecutive GGAA-repeats and higher $C A L C B$ expression levels in TC-71 EwS cells (12 repeats) as compared to MHH-ES1 EwS cells (9 repeats), we noted a higher enhancer activity of the GGAA-microsatellite derived from TC-71 as compared to the microsatellite derived from MHH-ES1 in luciferase assays (Fig. 2b, Supplementary Fig. S4). Collectively, these data provide evidence that $C A L C B$ is a direct EWSR1-FLI1 target gene, whose high but heterogeneous expression in EwS is regulated by EWSR1-FLI1 binding to an intronic, polymorphic, and enhancer-like GGAA-microsatellite.

\section{$C A L C B$ expression in primary EwS correlates with proliferation signatures}

To obtain first clues on the potential functional role of CALCB in EwS, we performed GSEA on CALCB coexpressed genes in a transcriptome dataset of 166 primary $\mathrm{EwS}^{32}$. GSEA revealed that $C A L C B$ is co-expressed with other EWSR1-FLI1 target genes (ZHANG_TARGETS_OF_EWSR1-FLI1_FUSION) ${ }^{37}$ and with gene signatures involved in stemness and proliferation (Fig. 3a, b).

CALCB signaling in EwS cells contributes to growth of EwS

To test the bioinformatic predictions from our GSEA in primary EwS, we carried out several functional experiments in EwS models. Mass spectrometric analysis showed that CALCB was readily detectable in FCS-free cell culture supernatants conditioned by A673 EwS cells, whereas it was not detectable in FCS-free cell culture medium not conditioned by EwS cells (Supplementary Table 2), suggesting that CALCB is indeed secreted by EwS cells. Notably, in accordance with the low expression levels of CALCA in primary EwS cells (Supplementary Fig. S1), CALCA was not detectable in cell culture supernatants of EwS cells (Supplementary Table 2).

To investigate the functional role of CALCB in EwS, we performed RNA interference experiments in two EwS cell lines (RDES and A673), which showed relatively high or moderate $C A L C B$ expression levels as compared to 20 other EwS cell lines (Supplementary Fig. S4). While the short-term knockdown of $C A L C B$ for 3 days had no effect on cellular proliferation (Supplementary Fig. S5), its longterm knockdown for 6-9 days significantly reduced proliferation and clonogenic growth in vitro (Fig. 4a, b). As no differences in the relative number of dead cells was detectable by Trypan-blue-labeled cell counting, the reduced cell count appeared to be not mediated by an increase in cell death (Fig. 4a).

Interestingly, knockdown of RAMP1-the crucial component of the CALCB receptor complex-phenocopied the effect of $C A L C B$ knockdown in clonogenic growth assays (Fig. 4b, Supplementary Fig. S6) as well as in 3D sphere-formation assays (Fig. 4c).

We next investigated whether knockdown of either gene could alter growth of xenografted EwS cells in vivo. To this end, we injected A673 cells, harboring a dox-inducible shRNA against either $C A L C B$ or $R A M P 1$, subcutaneously in NSG mice. When tumors were palpable, we induced the knockdown of the respective gene by addition of dox to the drinking water. In both settings, knockdown of the corresponding gene significantly 


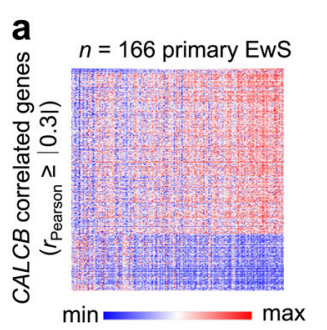

\begin{tabular}{ccccc} 
b & & & \\
& NES & $\boldsymbol{P}$ value & FDR $\boldsymbol{q}$ \\
\hline ZHANG_TARGETS_OF_EWSR1-FLI1_FUSION & 2.71 & $<0.001$ & $<0.001$ \\
& & & \\
WONG_EMBRYONIC_STEM_CELL_CORE & 2.71 & $<0.001$ & $<0.001$ \\
& 2.47 & $<0.001$ & $<0.001$
\end{tabular}

Fig. 3 CALCB expression in primary Ewing sarcoma (EwS) correlates with proliferation signatures. a Heatmap of CALCB correlated genes $\left(r_{\text {Pearson }} \geq|0.3|\right)$ in 166 primary EwS tumors. b Results of the gene set enrichment analysis on the ranked list of CALCB correlated genes as in Fig. $3 a$. NES normalized enrichment score

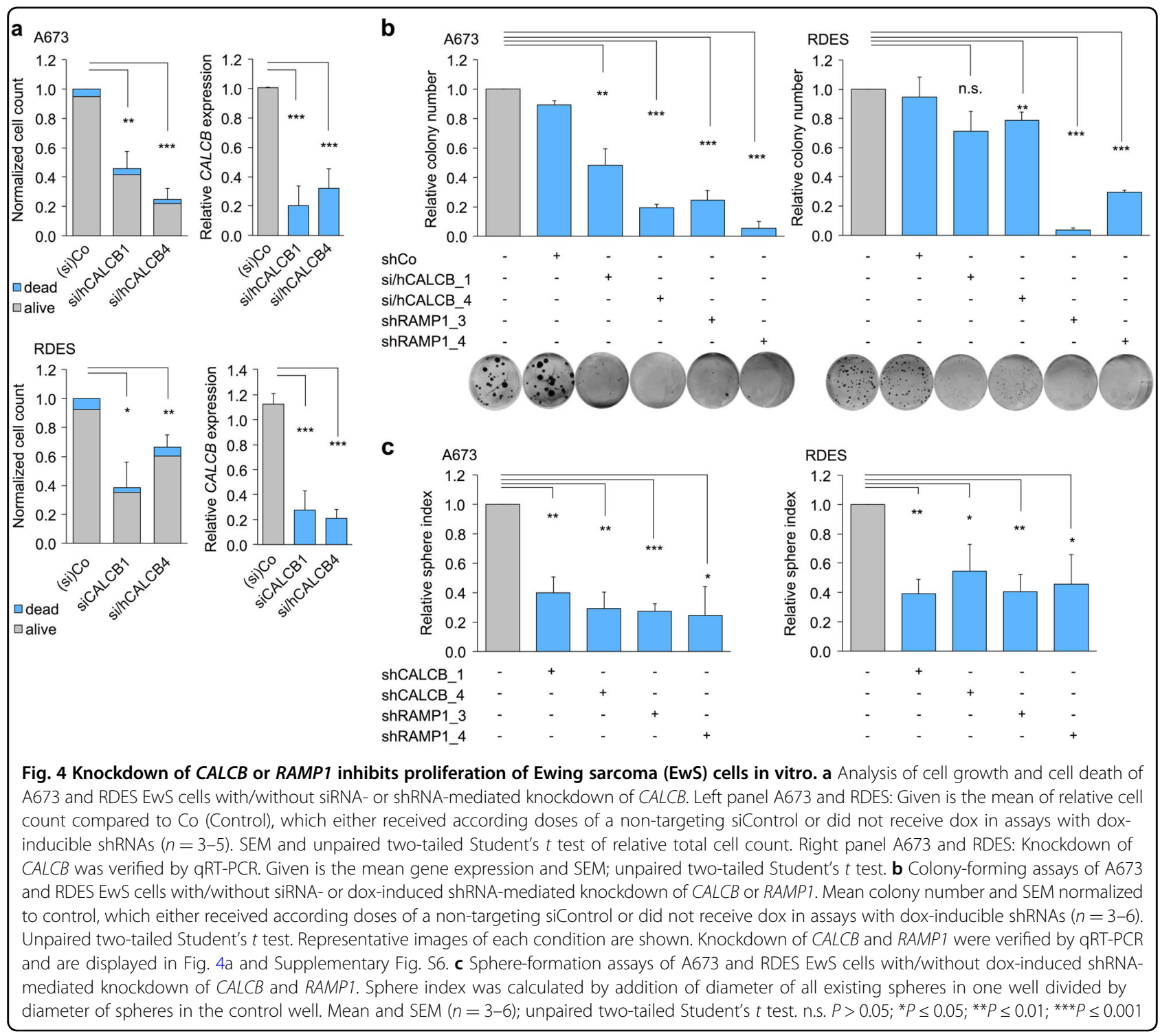

delayed tumor growth, which led to a delayed achievement of a mean tumor diameter of $15 \mathrm{~mm}$, the defined termination criteria, and therefore allowed a prolonged survival of the animals (Fig. 5a, b). However, dox treatment of mice carrying tumors with dox-inducible expression of a non-targeting control shRNA did not alter tumor growth compared to mice not receiving dox (data not shown), as also described previously for other 

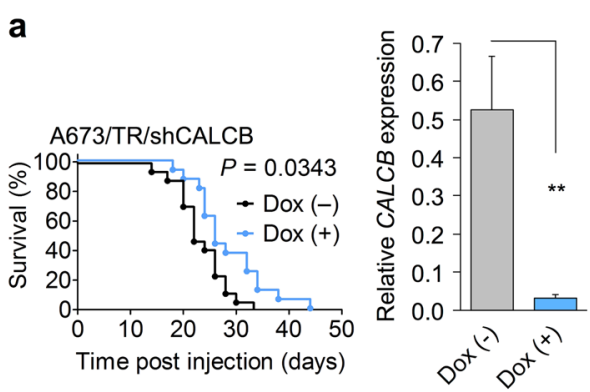

C

Xenografted $A 673$

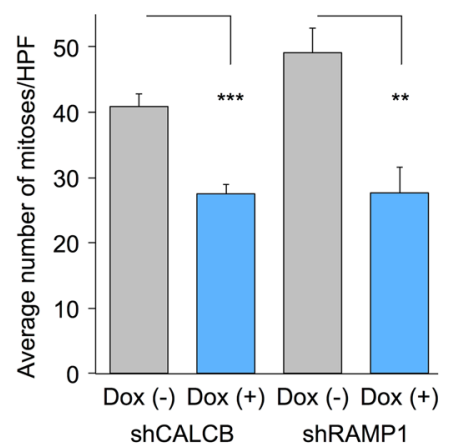

b
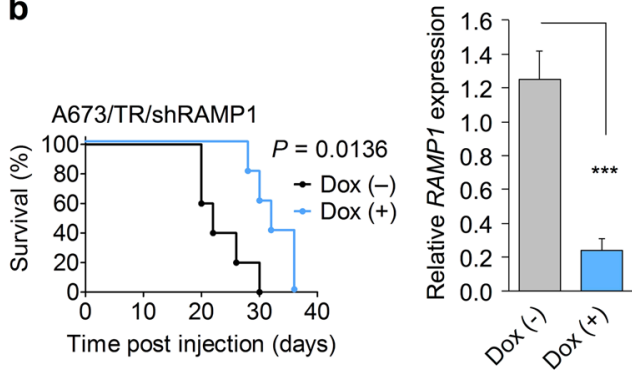

d

Xenografted A673

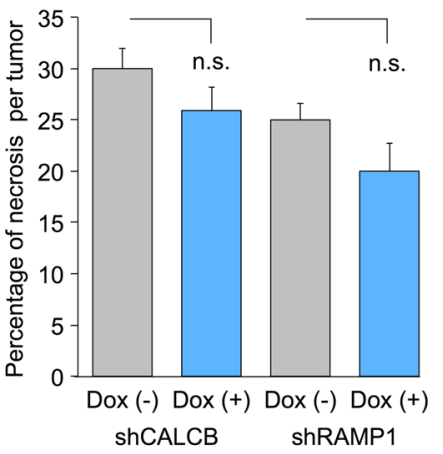

Fig. 5 Knockdown of CALCB or RAMP1 inhibits proliferation of Ewing sarcoma (EwS) cells in vivo. a Left panel: Analysis of tumor growth of A673 EwS cells with/without dox-induced knockdown of CALCB in NSG mice $(n=33)$. Event was defined as average diameter of $15 \mathrm{~mm}$. Event-free survival time of mice was analyzed by the Kaplan-Meier method and a log-rank test. Right panel: Knockdown of CALCB in the tumors of dox-treated mice was verified by qRT-PCR. Given are mean normalized gene expression levels and SEM; unpaired two-tailed Student's $t$ test. b Left panel: Analysis of tumor growth of A673 EwS cells with/without dox-induced knockdown of RAMP1 in NSG mice $(n=10)$. Event was defined as average diameter of $15 \mathrm{~mm}$. Eventfree survival time of mice was analyzed by the Kaplan-Meier method and a log-rank test. Right panel: Knockdown of RAMP1 in the tumors of dox-treated mice was verified by qRT-PCR. Given are mean normalized gene expression levels and SEM; unpaired two-tailed Student's $t$ test. c Histological analysis of the number of mitoses in tumor tissue of EwS xenografts with/without dox-induced knockdown of CALCB or RAMP1. Given is the mean number of mitoses and SEM per high-power filed (HPF) of 22 representative A673/TR/shCALCB xenografts shown in a and 10 A673/TR/shRAMP1 xenografts shown in b. Unpaired two-tailed, Student's $t$ test. $\mathbf{d}$ Histological analysis of necrosis in tumor tissue of EwS xenografts with/without dox-induced knockdown of CALCB or RAMP1. Given is the average percentage of necrotic area and SEM of 22 representative A673/TR/shCALCB xenografts shown in a and 10 A673/ TR/shRAMP1 xenografts shown in b. Unpaired two-tailed, Student's $t$ test. n.s. $P>0.05 ;{ }^{* *} P \leq 0.01 ;{ }^{* *} P \leq 0.001$

xenografted EwS cell lines ${ }^{31}$. Although $C A L C B$ and $R A M P 1$ were knocked down to low levels as confirmed by qRT-PCR of tumor tissue (Fig. 5a, b), the growthinhibiting effect was more pronounced in the group of the RAMP1 knockdown. Histological analysis of the xenografts revealed significantly higher mitotic activity in tumors without $C A L C B$ or RAMP1 knockdown, respectively, compared to tumors with shRNA-induced knockdown of either gene (Fig. 5c). In contrast, no differences in tumor necrosis was observed (Fig. 5d).

Taken together, these data suggest that CALCB is a secreted peptide in EwS and that the CALCB/RAMP1 axis promotes growth of EwS cells.

\section{Pharmacological inhibition of the CALCB/RAMP1 axis decreases growth of EwS cells}

To test whether the CALCB/RAMP1 axis could also be exploited therapeutically in EwS, we treated EwS cells with the small molecule CGRP receptor inhibitor MK-3207 for
3 days and quantified cell viability with a Resazurin assay. For these assays, we used dox-inducible $C A L C B$ or $R A M P 1$ knockdown EwS cells and applied increasing doses of MK3207. We observed a dose-dependent reduction of cell viability (Fig. 6a), which could be partially abrogated by knockdown of RAMP1-the central component of the inhibitor's target structure (Fig. 6b). These data suggest that, albeit relatively high doses of MK-3207 were applied to reduce viability of EwS cells, its effect was specific for the CALCB/RAMP1 axis. To validate these findings, we performed colony- and sphere-formation assays under MK3207 treatment and replicated these experiments with another small molecule CGRP inhibitor (Olcegepant, BIBN4096) (Fig. 6c, d). In both assays and for both inhibitors, we noted a significant reduction of 2D colony-formation and 3D sphere-formation capacity of EwS.

Together, these data provide further evidence for a functional role of the CALCB/RAMP1 axis in growth of EwS, which could potentially be exploited therapeutically. 

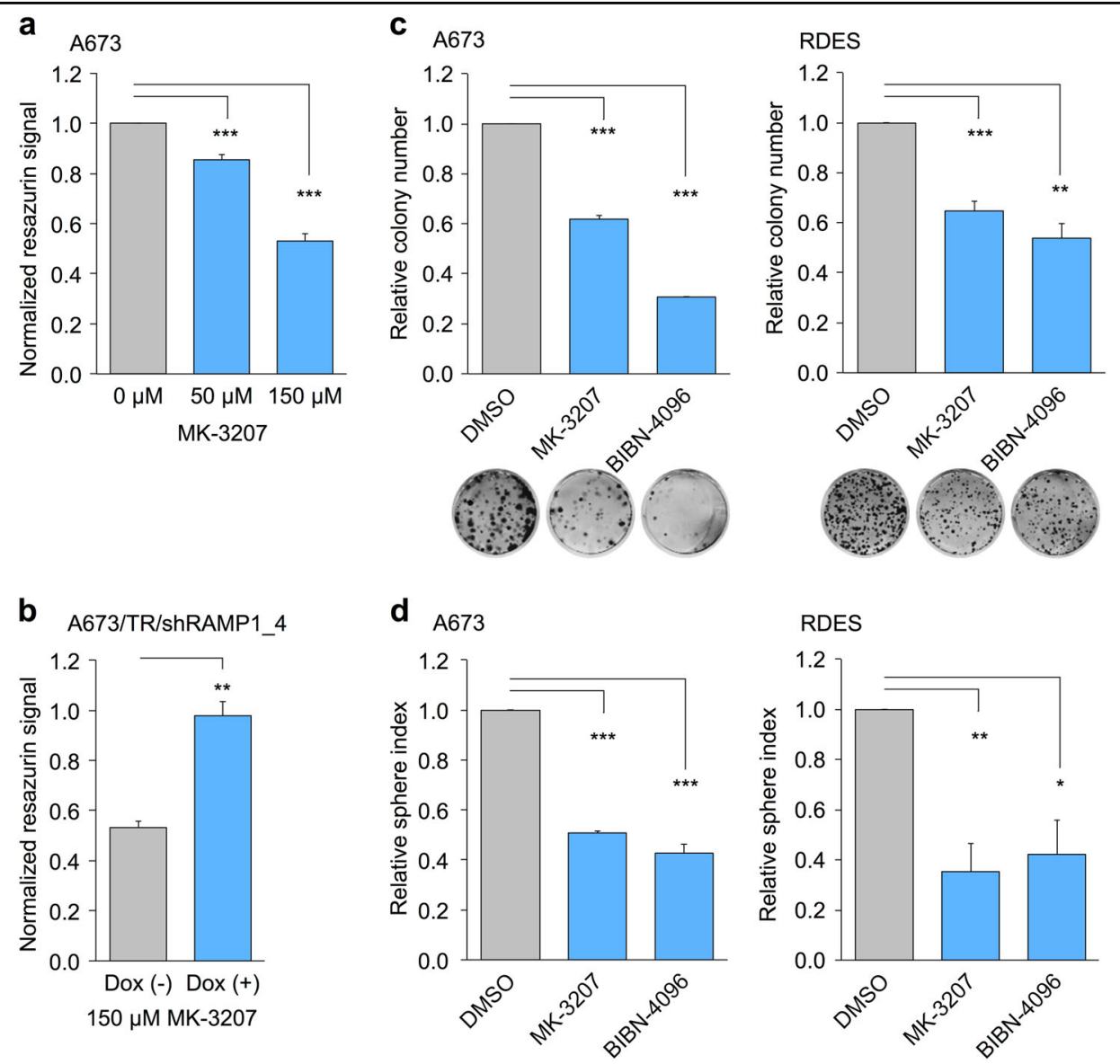

Fig. 6 Blockage of the calcitonin gene-related peptide (CGRP) receptor by small molecule inhibitors mimics the effect of CALCB and RAMP1 knockdown in vitro. a Analysis of cell viability (normalized Resazurin signal to DMSO control) in A673 Ewing sarcoma (EwS) cells treated for $72 \mathrm{~h}$ with the indicated concentrations of MK-3207. The graph shows the dose-dependent relative Resazurin signal. Data are represented as mean and SEM ( $n=12)$; unpaired two-tailed Student's $t$ test. $\mathbf{b}$ Comparison of relative Resazurin signal of A673 cells carrying a dox-inducible shRNA against RAMP1 treated with $150 \mu \mathrm{M}$ of MK-3207 with/without knockdown of RAMP1 by additional addition of $1 \mu \mathrm{g} / \mathrm{ml}$ dox to the growth medium. Data are represented as mean and SEM $(n=3)$; unpaired two-tailed Student's $t$ test. c Analysis of colony-forming capacity of A673 (left panel) and RDES (right

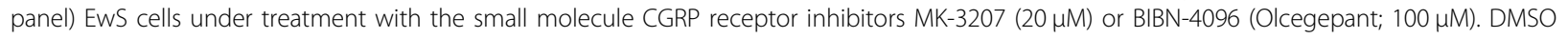
served as control for treatment. Representative images of the colonies are shown below. Data are represented as mean and SEM $(n=3)$; unpaired two-tailed Student's $t$ test. d Analysis of sphere-formation capacity of A673 (left panel) and RDES (right panel) EwS cells under treatment with the small molecule CGRP receptor inhibitors MK-3207 $(20 \mu$ M) or BIBN-4096 (Olcegepant; $100 \mu$ M). DMSO served as control for treatment. Sphere index was calculated by addition of diameters of all existing spheres in one well divided by diameter of spheres in the control well. Data are represented as mean and SEM $(n=3)$; unpaired two-tailed Student's $t$ test. ${ }^{*} P \leq 0.05 ;{ }^{* *} P \leq 0.01$; ${ }^{* *} P \leq 0.001$

\section{Discussion}

Albeit EwS is genetically well characterized, standard therapy still comprises only unspecific cytotoxic approaches. As it is not possible to directly target the action of EWSR1-ETS transcription factors, current strategies to find new and more specific treatment options include the investigation of target genes of EWSR1-ETS and determination of their potential as possible new therapy targets. To this end, we investigated the potential of the CALCB/RAMP1 axis as new target for treatment of EwS and explored its functional role in EwS cells by combining a series of in situ, in vitro, and in vivo experiments. We found that CALCB is a secreted peptide in EwS that shows a highly specific expression pattern among malignant and normal tissues. The high expression of $C A L C B$ in EwS is likely driven by EWSR1-FLI1 binding to a GGAAmicrosatellite at the $C A L C B$ locus. Since this GGAAmicrosatellite is transformed into a de novo enhancer upon EWSR1-FLI1 binding but does not show enhancer activity in the absence of EWSR1-FLI1, we speculate that different mechanisms may operate in other tissue types such as trigeminal ganglia to upregulate $C A L C B$ expression, which remain to be elucidated.

In our long-term knockdown experiments, we observed that silencing of $C A L C B$ or RAMP1 reduced growth of EwS cells in vitro and in vivo. To the best of our knowledge, this is the first report of a functional role of CALCB in growth of cancer cells to date. However, we noted a weaker effect of 
the CALCB knockdown as compared to that of RAMP1 on tumor growth in in vivo experiments, which may be caused by residual $C A L C B$ expression in the EwS cells (around 5\% remaining expression), or alternatively by circulating murine Calcb, which might have compensated at least in part for the loss of human CALCB.

In our drug-response assays, we found that inhibition of CGRP receptors with two different small molecules had a similar antiproliferative effect on EwS cells to that of the CALCB or RAMP1 knockdown. CGRP receptor inhibitors already showed high efficacy in the treatment of migraine, which is presumably caused via CALCA- or CALCBmediated vasodilatation in the vicinity of the trigeminal ganglia-the only normal tissue type with physiologically high $C A L C B$ expression levels found in our analyses. We speculate that repurposing and further optimization of such "migraine drugs" (e.g., newly developed antibodies directed against CALCB or RAMP1) ${ }^{38}$ could perhaps offer novel therapeutic options for EwS patients in the future. As we did not observe differences in density of tumorassociated blood vessels quantified by staining for murine CD31 in IHC of our xenograft experiments (Supplementary Fig. S7), we assume that the growth-promoting effect of the CALCB/RAMP1 axis is conferred via different mechanisms than one would expect from the vasodilatory effect of CALCB known from the literature ${ }^{39}$.

Collectively, we identified $C A L C B$ as a highly specifically expressed EWSR1-FLI1 target gene encoding a secreted peptide that promotes growth of EwS cells, and show that targeting the CALCB/RAMP1 axis in EwS may offer a new therapeutic approach. Future studies will have to dissect the precise downstream signaling and how the CALCB/RAMP1 axis promotes proliferation of EwS cells to further explore its therapeutic potential.

\section{Acknowledgements}

We thank A. Sendelhofert and A. Heier for excellent technical assistance and P. Gilardi-Hebenstreit for technical advice. The laboratory of T.G.P.G. is supported by grants from the "Verein zur Förderung von Wissenschaft und Forschung an der Medizinischen Fakultät der LMU München (WiFoMed)," by LMU Munich's Institutional Strategy LMUexcellent within the framework of the German Excellence Initiative, the "Mehr LEBEN für krebskranke Kinder - Bettina-BräuStiftung," the Walter Schulz Foundation, the Wilhelm Sander-Foundation (2016.167.1), the Friedrich-Baur foundation, the Matthias-Lackas foundation, the Dr. Leopold und Carmen Ellinger foundation, the Gert \& Susanna Mayer foundation, the Deutsche Forschungsgemeinschaft (DFG 391665916), and by the German Cancer Aid (DKH-111886 and DKH-70112257). J.L. was supported by a scholarship of the China Scholarship Council (CSC). J.M. was supported by a scholarship of the "Kind-Philipp-Foundation," M.D. by a scholarship of the "Deutsche Stiftung für Junge Erwachsene mit Krebs," and T.L.B.H. by a scholarship of the German Cancer Aid. M.C.B., M.F.O., and M.M.L.K. were supported by scholarships of the German National Academic Foundation. M.C.B. was supported by a scholarship of the Max Weber-Program of the State of Bavaria.

\footnotetext{
Author details

${ }^{1}$ Max-Eder Research Group for Pediatric Sarcoma Biology, Institute of Pathology of the LMU Munich, Munich, Germany. Institute of Pathology of the LMU Munich, Munich, Germany. ${ }^{3}$ German Cancer Consortium (DKTK), Heidelberg, Germany. ${ }^{4}$ German Cancer Research Center (DKFZ), Heidelberg, Germany. ${ }^{5}$ Department of Medicine, Division of Infectious Diseases, and IIRC
}

Antibody Engineering and Proteomics facility, University of British Columbia, Vancouver, BC, Canada

Conflict of interest

The authors declare that they have no conflict of interest.

\section{Publisher's note}

Springer Nature remains neutral with regard to jurisdictional claims in published maps and institutional affiliations.

Supplementary Information accompanies this paper at (https://doi.org/ 10.1038/s41419-019-1372-0).

Received: 9 December 2018 Revised: 16 January 2019 Accepted: 17 January 2019

\section{References}

1. Grünewald, T. G. P. et al. Ewing sarcoma. Nat. Rev. Dis. Prim. 4, 5 (2018).

2. Ladenstein, R. et al. Primary disseminated multifocal Ewing sarcoma: results of the Euro-EWING 99 trial. J. Clin. Oncol. 28, 3284-3291 (2010).

3. Delattre, O. et al. Gene fusion with an ETS DNA-binding domain caused by chromosome translocation in human tumours. Nature 359, 162-165 (1992).

4. Anderson, N. D. et al. Rearrangement bursts generate canonical gene fusions in bone and soft tissue tumors. Science 361, https://doi.org/10.1126/science. aam8419 (2018).

5. Gangwal, K. et al. Microsatellites as EWS/FLI response elements in Ewing's sarcoma. Proc. Natl Acad. Sci. USA 105, 10149-10154 (2008).

6. Tomazou, E. M. et al. Epigenome mapping reveals distinct modes of gene regulation and widespread enhancer reprogramming by the oncogenic fusion protein EWS-FLI1. Cell Rep. 10, 1082-1095 (2015).

7. Riggi, N. et al. EWS-FLI1 utilizes divergent chromatin remodeling mechanisms to directly activate or repress enhancer elements in Ewing sarcoma. Cancer Cell 26, 668-681 (2014).

8. Tirode, F. et al. Genomic landscape of Ewing sarcoma defines an aggressive subtype with co-association of STAG2 and TP53 mutations. Cancer Discov. 4, 1342-1353 (2014).

9. Crompton, B. D. et al. The genomic landscape of pediatric Ewing sarcoma. Cancer Discov. 4, 1326-1341 (2014).

10. von Levetzow, C. et al. Modeling initiation of Ewing sarcoma in human neural crest cells. PLOS ONE 6, e19305 (2011).

11. Tirode, F. et al. Mesenchymal stem cell features of Ewing tumors. Cancer Cell. 11, 421-429 (2007).

12. Minas, T. Z. et al. Combined experience of six independent laboratories attempting to create an Ewing sarcoma mouse model. Oncotarget 8 , 34141-34163 (2017).

13. Lambert, M., Jambon, S., Depauw, S. \& David-Cordonnier, M-H. Targeting transcription factors for cancer treatment. Molecules 23, https://doi.org/ 10.3390/molecules23061479 (2018).

14. Höppener, J. W. et al. Expression of the second calcitonin/calcitonin generelated peptide gene in Ewing sarcoma cell lines. J. Clin. Endocrinol. Metab. 64, 809-817 (1987).

15. Steenbergh, P. H., Höppener, J. W., Zandberg, J., Lips, C. J. \& Jansz, H. S. A second human calcitonin/CGRP gene. FEBS Lett. 183, 403-407 (1985).

16. Hoovers, J. M. et al. High-resolution chromosomal localization of the human calcitonin/CGRP/IAPP gene family members. Genomics 15, 525-529 (1993).

17. Steenbergh, P. H. et al. Structure and expression of the human calcitonin/ CGRP genes. FEBS Lett. 209, 97-103 (1986).

18. Wimalawansa, S. J. Calcitonin gene-related peptide and its receptors: molecular genetics, physiology, pathophysiology, and therapeutic potentials. Endocr. Rev. 17, 533-585 (1996).

19. Wimalawansa, S. J, Morris, H. R. \& Maclntyre, I. Both alpha- and beta-calcitonin gene-related peptides are present in plasma, cerebrospinal fluid and spinal cord in man. J. Mol. Endocrinol. 3, 247-252 (1989).

20. McLatchie, L. M. et al. RAMPs regulate the transport and ligand specificity of the calcitonin-receptor-like receptor. Nature 393, 333-339 (1998).

21. Hay, D. L. What makes a CGRP2 receptor? Clin. Exp. Pharmacol. Physiol. 34 963-971 (2007) 
22. van Valen, F., Keck, E. \& Jürgens, H. Functional characteristics of calcitonin gene-related peptide receptors in human Ewing's sarcoma WE-68 cells. FEBS Lett. 256, 170-174 (1989).

23. Baldauf, M. C. et al. Systematic identification of cancer-specific MHC-binding peptides with RAVEN. Oncoimmunology 7, e1481558 (2018).

24. Dai, M. et al. Evolving gene/transcript definitions significantly alter the interpretation of GeneChip data. Nucleic Acids Res. 33, e175 (2005).

25. Irizarry, R. A. et al. Exploration, normalization, and summaries of high density oligonucleotide array probe level data. Biostatistics 4, 249-264 (2003).

26. Grünewald, T. G. P. et al. STEAP1 is associated with the invasive and oxidative stress phenotype of Ewing tumors. Mol. Cancer Res. 10, 52-65 (2012).

27. Carrillo, J. et al. Cholecystokinin down-regulation by RNA interference impairs Ewing tumor growth. Clin. Cancer Res. 13, 2429-2440 (2007).

28. Livak, K. J. \& Schmittgen, T. D. Analysis of relative gene expression data using real-time quantitative PCR and the 2(-Delta Delta C(T)) Method. Methods 25, 402-408 (2001).

29. Machiela, M. J. et al. Genome-wide association study identifies multiple new loci associated with Ewing sarcoma susceptibility. Nat. Commun. 9, 3184 (2018).

30. ENCODE Project Consortium. An integrated encyclopedia of DNA elements in the human genome. Nature 489, 57-74 (2012).

31. Grünewald, T. G. P. et al. Chimeric EWSR1-FLI1 regulates the Ewing sarcoma susceptibility gene EGR2 via a GGAA microsatellite. Nat. Genet. 47, 1073-1078 (2015).
32. Baldauf, M. C. et al. Robust diagnosis of Ewing sarcoma by immunohistochemical detection of super-enhancer-driven EWSR1-ETS targets. Oncotarget 9, 1587-1601 (2018)

33. Subramanian, A. et al. Gene set enrichment analysis: a knowledge-based approach for interpreting genome-wide expression profiles. Proc. Natl Acad. Sci. USA 102, 15545-15550 (2005).

34. Wiederschain, D. et al. Single-vector inducible lentiviral RNAi system for oncology target validation. Cell Cycle 8, 498-504 (2009).

35. Remmele, W. \& Stegner, H. E. [Recommendation for uniform definition of an immunoreactive score (IRS) for immunohistochemical estrogen receptor detection (ER-ICA) in breast cancer tissue]. Pathologe 8, 138-140 (1987).

36. Hansen, $\mathrm{S}$. et al. The prognostic value of angiogenesis by Chalkley counting in a confirmatory study design on 836 breast cancer patients. Clin. Cancer Res. $\mathbf{6}$, 139-146 (2000).

37. Hu-Lieskovan, S. et al. EWS-FLI1 fusion protein up-regulates critical genes in neural crest development and is responsible for the observed phenotype of Ewing's family of tumors. Cancer Res. 65, 4633-4644 (2005).

38. Edvinsson, L., Haanes, K. A., Warfvinge, K. \& Krause, D. N. CGRP as the target of new migraine therapies - successful translation from bench to clinic. Nat. Rev. Neurol. 14, 338-350 (2018).

39. Tippins, J. R. CGRP: a novel neuropeptide from the calcitonin gene is the most potent vasodilator known. J. Hypertens. Suppl. 4, S102-105 (1986).

40. Gordon, D. J., Motwani, M. \& Pellman, D. Modeling the initiation of Ewing sarcoma tumorigenesis in differentiating human embryonic stem cells. Oncogene 35, 3092-3102 (2016). 